\title{
Histological Observation and Expression Patterns of antimicrobial peptides during Fungal Infection in Musca domestica (Diptera: Muscidae) Larvae
}

\author{
Xiu Jiangfan', Wang Tao ${ }^{1}$, Wang Yu ${ }^{1,2}$, Wu Jianwei ${ }^{1,3 *}$, Guo Guo', Zhang Yingchun ${ }^{1}$, \\ Shang Xiaoli ${ }^{3}$ \\ ${ }^{1}$ School of Basic Medical Sciences, Guizhou Medical University, Guiyang 550004, China; ${ }^{2}$ Guizhou Provincial \\ Center for Disease Control and Prevention, Guiyang 550004, China; ${ }^{3}$ School of Biology \& Engineering, Guizhou \\ Medical University, Guiyang 550004, China.
}

\begin{abstract}
Housefly, Musca domestica, has a complicated immune system. However, its underlying operating mechanism remains elusive. Candida albicans is a major pathogen affecting humans by causing deep infection fungous disease, but it is non-symbiotic in houseflies. To investigate the $C$. albicans infection process in housefly, the changes in morphological and histological and expression patterns of antimicrobial peptide were monitored to indicate the insect's response to fungal infection. The results showed that scattered brown spots were comprising melanized encapsulation and encapsulated fungal cells were initially observed at the inner side of larvae's body wall $3 \mathrm{~h}$ postinfection (PI). Between 6 and $36 \mathrm{~h} \mathrm{PI}$, the whole body of larvae was densely covered with the brown spots, which then gradually disappeared. The majority had disappeared at $48 \mathrm{~h}$ PI. Some fungi colonized in the gaps between the body wall and the muscle layer, as well as among muscle fibers of the muscle layer at $12 \mathrm{~h}$ PI and hyphal was observed at $18 \mathrm{~h}$ PI. These fungi colonized distribution changed from a continuous line to scattered spots at $24 \mathrm{~h}$ PI and virtually disappeared at $48 \mathrm{~h}$. The results of quantitative PCR analysis revealed that in coordination with the variation during the infection, the expression levels of four antimicrobial peptides were up-regulated. In conclusion, $C$. albicans infection in $M$. domestica larvae involved the following stages: injection, infection, immune response and elimination of the pathogen. The rapid response of antimicrobial peptides, melanized encapsulation and agglutination played a vital role against the pathogenic invasion.
\end{abstract}

Key words: Musca domestica, larvae, Candida albicans, infection, innate immunity

*Authors for correspondence:jianweiwu519@sina.com 


\section{INTRODUCTION}

Insects lack the adaptive immune responses typical of vertebrates; thus, they are heavily dependent on their innate immune system to guard against pathogen invasion (Hoffmann 2003). Vector insects, with their susceptibility to human pathogens, which can carry a great variety of pathogens and cause many diseases, hence lead to serious damages to human health, and their immune response mechanism is highly homologous to the mammalian innate immune response (Cirimotich et al. 2011; Stokes et al. 2015). In what way the insect innate immune system responds to human pathogens has become a recent focus of research. Many studies have investigated the host-pathogen interaction between insects and pathogen; for example, the interaction between the insects Galleria mellonella, Drosophila melanogaster, silkworm and Tribolium castaneum and the pathogens Candida albicans, Cryptococcus neoformans, Pseudomonas aeruginosa, Escherichia coli, Staphylococcus aureus and Streptococcus pneumoniae (Altincicek et al. 2008; Fuchs et al. 2010; Chamilos et al. 2011; Wang et al. 2013). Recent research indicates that the insect immune system can be activated by the alien pathogens. When the insects are infected by the invading pathogens, the pattern recognition proteins/receptor (PRPs) in insects would firstly recognize and combine with pathogen-associated molecular pattern (PAMPs) in the pathogens, and then initiate the activation and regulation of a series of innate immune response (Ohta et al. 2006). On the one hand, the hemolymph cell served as the major cellular immune response triggering cytophagocytosis and melanization tubercle formation (Lavine and Strand 2002; Lu 2008; Wu et al. 2015). On the other hand, immune effector molecules such as antimicrobial peptides (AMPs) and lysozymes synthesized and secreted by fat body cells (i.e. similar to mammals' liver), hemocytes and other cells trigger humoral immune responses (Lemaitre and Hoffmann 2007). The innate immune response in insects is divided into humoral immunity and cellular immunity. They function together to kill and eliminate the pathogens via phagocytosis, nodulation, encapsulation, coagulation, and melanization.

Houseflies are present worldwide and considered to be an important medical insect which can carry and transmit over 100 human pathogens and zoonotic agents, but they rarely to be infected (Scott et al. 2014). It has been demonstrated that housefly larvae were prone to induce the generation of AMPs such as attacin, cecropin, defensin and diptericin by means of heat shock, ultraviolet exposure and needle piercing (Wang et al. 2006; Liang et al. 2006; Dang et al. 2010; Liu et al. 2011). Once the adult housefly is infected with Beauveria bassiana, hemocyte density changes and fungi number in the hemolymph are synchronized (Mishra et al. 2015). The published genome sequences analysis of housefly indicated that compared with Drosophila melanogaster, the genome contains a rich resource of shared and novel protein coding genes, a significantly higher amount of repetitive elements, and substantial increases in copy number and diversity of both the recognition and effector components of the immune system, including a large number of genes encoding PAMPs, immunerelated signalling pathways, immune effector molecules and antimicrobial peptides (Scott et al. 2014). Moreover, many specific peptides with strong antibacterial effects are now being discovered in houseflies, including MAF-1, Muscin and MDAP-2 (Fu et al. 2009, 2015; Pei et al. 2014; Yang et al. 2015). These results indicate that houseflies are gifted with a complicated innate immune system that has likely adapted to the complex living environment, particularly to the pathogens these houseflies carry and transmit. However, the mechanisms underlying the operation of this unique innate immune system remains elusive, and the interactions between houseflies and pathogens require further studies.

C. albicans is an opportunistic fungus that can cause deep fungal infections and severely harm human health (Miceli et al. 2011). However, it is an exogenous and non-symbiotc fungus in a housefly (Phoku et al. 2014), furthermore, there was no direct evidence that Candida albicans is a natural pathogen of $M$. domestica. In our preliminary study, all the larvae which injected with $210 \mathrm{nl}$ PBS buffer were able to complete their life cycle, and in the meanwhile, the larvae injected with an equal volume of the fugal suspension (i.e. approximately $2 \times 10^{4} \mathrm{CFU}$ of $C$. albicans) were controlled with the ratio of approximately $50 \%$. On the basis of predecessors study, it provides a good opportunity to investigate the interrelation between an exogenous fungus and the innate immune system of a housefly during the period of infection and recovery. Therefore, we sought to describe the 
infection process and broaden the understanding of the insect's immune response to fungal infections.

\section{MATERIAL AND METHODS}

\section{House fly rearing}

Houseflies were bred in the Modern Pathogenic Biology Laboratory, Guizhou Medical University (Guiyang, China) (Fu et al. 2009). Their larvae were raised on an artificial diet comprising bran and water and routinely reared at $26-28^{\circ} \mathrm{C}$ with $70-80 \%$ relative humidity and a photoperiod of $12 \mathrm{~h}: 12$ (Light: Dark) h for up to the third-instar larval stage.

\section{C. albicans culturing}

The Candida albicans (ATCC10231) used in infection experiments was stored at our laboratory. C. albicans was cultivated in Sabouraud dextrose agar (SDA) and its cell suspension was prepared by inoculating a single colony in Sabouraud dextrose broth (SDB) at $37^{\circ} \mathrm{C}$ for $12 \mathrm{~h}$ with agitation (Kelly and Kavanagh 2011). Fungus entering the logarithmic growth phase was selected for infection experiments. $C$. albicans cells solution were centrifuged at $4000 \mathrm{~g} / \mathrm{min}$ and washed and resuspended in phosphate buffered saline $(1 \times$ PBS $0.01 \mathrm{M}$, Solarbio, China) and standardized to $1 \times 10^{8}$ $\mathrm{CFU} / \mathrm{ml}$.

\section{Infection experiments}

Each experimental group comprised 200 randomly chosen third-instar larvae of appropriate weight (26 $\pm 0.5 \mathrm{mg}$ ). The infected group (IC group) larvae were injected with $210 \mathrm{nl}$ suspension, i.e. approximately $2 \times 10^{4} \mathrm{CFU}$ of $C$. albicans at the 10th segment of segmental venter of larvae using the Auto-Nanoliter Injector (Nanoject II Nanoliter Injector, Drummond Scientific Co., Broomall, USA) under a stereomicroscope (SMZ25, Nikon, Japan) (Khalil et al. 2015). After injection, these larvae were raised on sterilized bran and water. At $3,6,12,18,24,36$ and $48 \mathrm{~h}$ post-infection (PI), the larvae were collected. Untreated larvae were also collected (blank control group, C group), and larvae injected with an equal volume of PBS buffer were used as the negative control group (PBS group).

Morphological and histological observations Larvae were collected at the indicated time points. Morphology was observed using a stereoscopic microscope (SMZ25, Nikon, Japan). After embedding in Tissue-Tek optimal cutting temperature compound (OCT, Sakura Finetek, Netherlands), the larvae were transferred to a microtome cryostat (MEV3.01, SLEE, Germany). Histological sections of $10 \mu \mathrm{m}$ were removed and subjected to periodic acid Schiff (PAS) staining (Okada et al. 2013), observed and photographed using a microscope (Eclipse Ci-S, Nikon, Japan) and NIS-Elements System (NIS-Elements, Nikon Instruments Inc., USA).

\section{qPCR analyses of AMP genes expression}

Total RNA was isolated from 6-8 larvae using TRIzol $^{\circledR}$ Reagent (TRIzol ${ }^{\circledR}$ Reagent, Invitrogen, USA) strictly following the manufacturer's protocol. The concentration and purity level of samples was detected by NanoDrop (ND1000, Thermo Scientific, USA) and agarose (1\%) gel electrophoresis. Then, $1 \mu \mathrm{g}$ of total RNA was reverse-transcribed in $10 \mu$ reaction volumes using PrimeScript RT (PrimeScriptTM RT reagent Kit with gDNA Eraser, Takara Bio, Japan) with random hexamer and oligo dT primers. After synthesis, cDNA was diluted 1: 10 and used in quantitative polymerase chain reaction (qPCR) analysis. Each reaction was performed using the SYBR Premix (SYBR Premix Ex TaqTM II, Takara Bio, Japan), and reactions were performed using the real-time PCR system (ABI PROSM 7300, Applied Biosystems, USA). Reaction mixtures were incubated for $30 \mathrm{~s}$ at $95^{\circ} \mathrm{C}$, followed by 40 cycles of $5 \mathrm{~s}$ at $95^{\circ} \mathrm{C}$ and $34 \mathrm{~s}$ at $60^{\circ} \mathrm{C}$. Primers for attacin, cecropin, defensin, diptericin and reference (rps18) genes were selected on the basis of previous studies on housefly immune responses (Wang et al. 2009; Fleming et al. 2014). The primers were designed using Primer 5.0 software (Primer PREMIER Version 5.00, PREMIER Biosoft International 2000). The primers used for $q P C R$ are listed in Table 1. For relative expression analysis, CT values for calibrator conditions (i.e. larvae untreated at each indicated time points, $C$ group) and treatment groups (i.e. PBS and IC groups) were calculated using the $2^{-\Delta \Delta C T}$ method (Pfaffl et al. 2002). $\log _{2^{-}}$ fold change in the expression ratios of the target genes attacin, cecropin, defensin and diptericin were compared with the calibrator condition using the reference gene rps18. Statistical significance difference in the IC and PBS groups were analyzed by multiple $t$-test using the Holm-Sidak method (PASW Statistics 18, SPSS Inc. 2010). All data was 
analysed to determine differences between groups $(P<0.05)$.

Table 1 Primer sequences for qPCR

\begin{tabular}{llll}
\hline Gene & Access no. & Sense Sequence (5'-3') & Antisense Sequence (5'-3') \\
\hline rps18 & KC424479.1 & GCGTGACGATTTGGAACGCTTGA & TTCTTGGATACACCGACAGTGCGA \\
attacin & DQ062744.1 & CGAATTCATGTTCTCTAAATC & CTCTAGATTAAAAATCATGACTT \\
cecropin & ES608437.1 & GGACAAAGTGAAGCTGGATGGTTG & GCTGGGCCACACCAATAGTTTGA \\
defensin & ES608345.1 & AAATTTCGTCCATGGAGCTGACGC & ACCGCTCAACAAATCGCAAGTAGC \\
diptericin & ES608652.1 & AGTGCAACATTTGTGGTTGCCGAC & GCCATAACCTGCTGTGGCATCA \\
\hline
\end{tabular}

\section{RESULTS}

\section{Morphological observations}

Morphological observations revealed no changes under the epidermis of the larvae in the $\mathrm{C}$ and PBS groups (Fig. 1A, Fig. 1B). However, under the body wall of the larvae in the IC group, many scattered brown spots were observed PI. At $3 \mathrm{~h}$ PI these spots were scattered and distributed at the inner side of larvae's body wall (Fig. 1C), and at $6 \mathrm{~h}$ PI, the spots had substantially increased in both number and size (Fig. 1D). At 12, 18 and $24 \mathrm{~h} \mathrm{PI}$, the entire body was densely covered by brown spots of various sizes (Figs. 1E, 1F and 1G). At $36 \mathrm{~h} \mathrm{PI}$, the quantity of these spots had significantly decreased and their colour had faded (Fig. 1H). Majority of the spots had disappeared at $48 \mathrm{~h}$ PI (Fig. 1I).
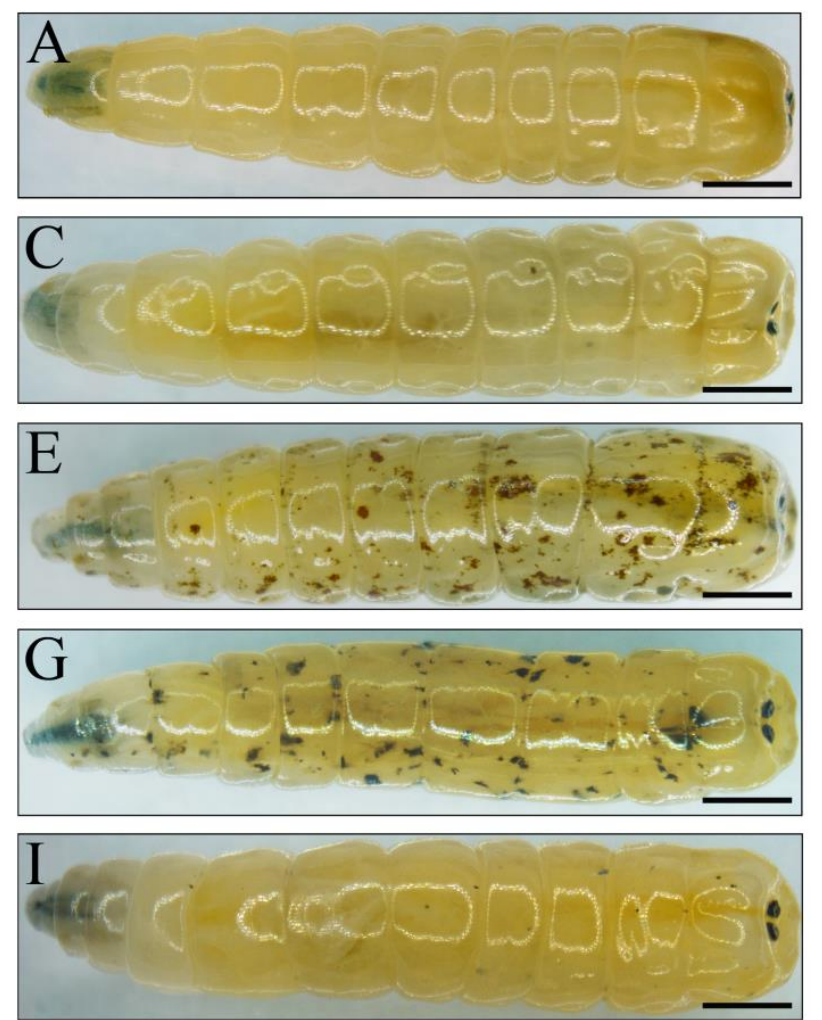

Figure 1 Morphology observation of housefly larvae after Candida albicans injection. A Blank control group: untreated larvae. B PBS negative control: larvae were injected with $210 \mathrm{nl}$ PBS buffer. C-I Infection group: the larvae were injected with $210 \mathrm{nl}$ suspension of $C$. albicans $\left(2 \times 10^{4} \mathrm{CFU}\right)$. C $3 \mathrm{~h}$ post infection (PI), D $6 \mathrm{~h}$ PI, E 12 h PI, F 18 h PI, G 24 h PI, H 36 h PI and I 48 h PI. The arrow indicated the brown spots under the body wall of the larvae. $(\mathrm{A}-\mathrm{I})$ Scale $\mathrm{Bar}=1.0 \mathrm{~mm}$.

\section{Histological observations}

We performed histological studies to better observe C. albicans infection process in the housefly. Periodic Acid Schiff (PAS) staining could clearly distinguish larvae tissue and fungi; tissue was stained light or dark blue, whereas $C$. albicans was stained purple red. There were no differences between the larvae in the $\mathrm{C}$ and PBS groups (Figs. $2 \mathrm{~A}$ and $3 \mathrm{~A}$, Figs. $2 \mathrm{~B}$ and $3 \mathrm{~B}$ ). We observed a certain amount of $C$. albicans cells distributed in the gaps between the body wall and the muscle layer, as well as among muscle fibers of the muscle layer of infected larvae in the IC group. At 3 and $6 \mathrm{~h} \mathrm{PI}$, considerable aggregation of $C$. albicans cells (yeast forms) distributed in these gaps (Figs. 2C and 3C, Figs. 2D and 3D). At $12 \mathrm{~h} \mathrm{PI}$, fungal cells were observed colonization in a linear arrangement in these gaps between the body wall and the muscle layer and among muscle fibers of the muscle layer. The growth of the fungus and its filamentation led to thses tissues invasion, some of them were observed with short hyphae (Figs. 2E and 3E). At $18 \mathrm{~h} \mathrm{PI}$, the distribution of fungal changed from a continuous line to scattered spots, and a significant number of $C$. albicans with hyphae and yeast forms were observed (Figs. 2F and 3F). At $24 \mathrm{~h}$ PI, majority of the $C$. albicans had hyphae (Figs. $2 \mathrm{G}$ and $3 \mathrm{G})$. At $36 \mathrm{~h} \mathrm{PI}$, the number of $C$. albicans had significantly decreased; furthermore, $C$. albicans with hyphal forms were sparsely dotted in the muscle tissue (Figs. $2 \mathrm{H}$ and $3 \mathrm{H}$ ) and had virtually disappeared at $48 \mathrm{~h} \mathrm{PI} \mathrm{(Figs.} \mathrm{2I} \mathrm{and} \mathrm{3I).}$ 

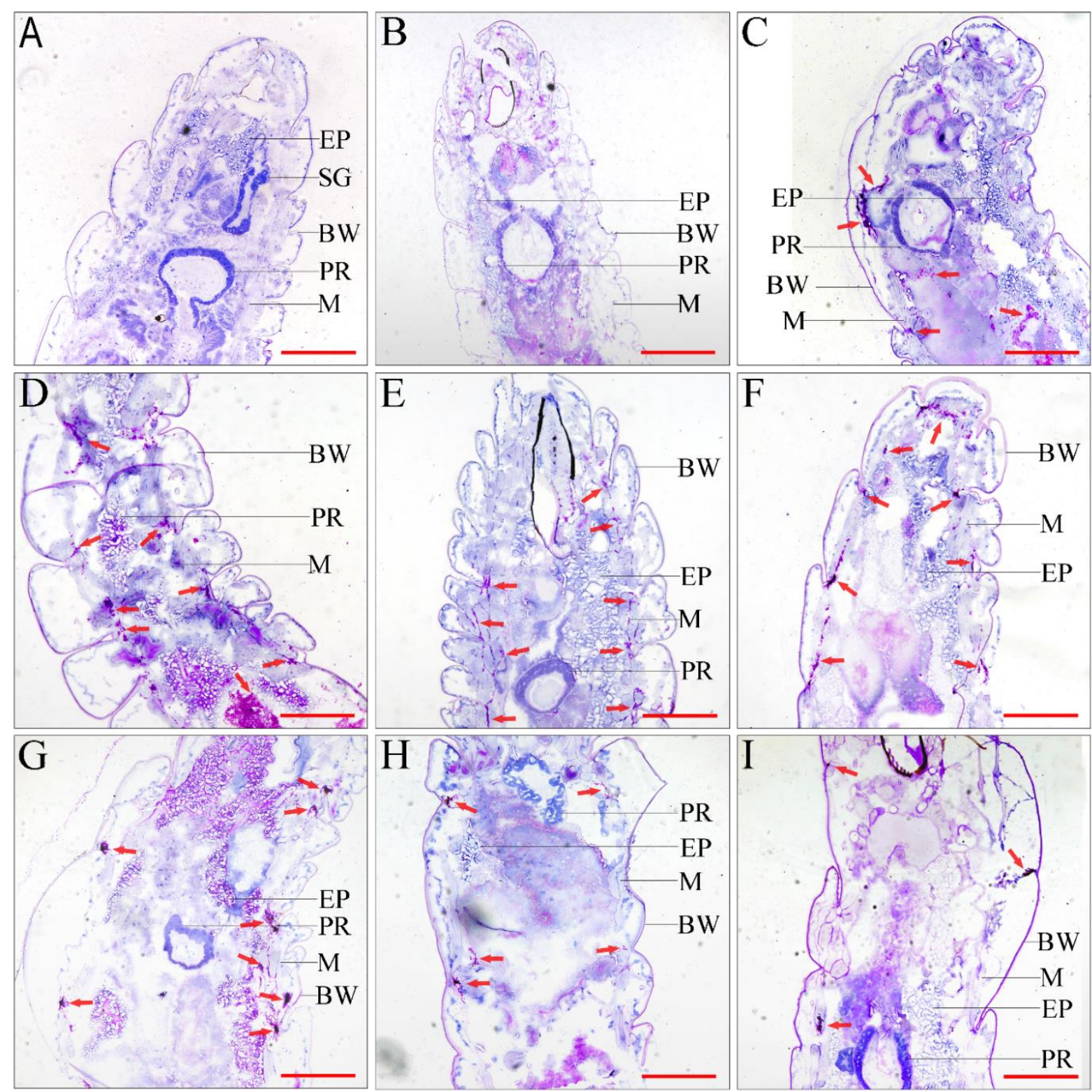

Figure 2 Histopathological identification of $C$. albicans in housefly larvae (PAS stain, $\times 40$ ). A Blank control group: untreated larvae. B PBS negative control: larvae were injected with equal volume PBS buffer. C-I Infection group: larvae were injected with C. albicans larvae. C 3 h post-infection (PI), D 6 h PI, E 12 h PI, F 18 h PI, G 24 h PI, H $36 \mathrm{~h}$ PI and I 48 h PI. Proventriculus (PR), salivary glands (SG), body wall (BW), epiploon (EP), muscle (M), arrows indicate C. albicans. Bar $=500 \mu \mathrm{m}$ 


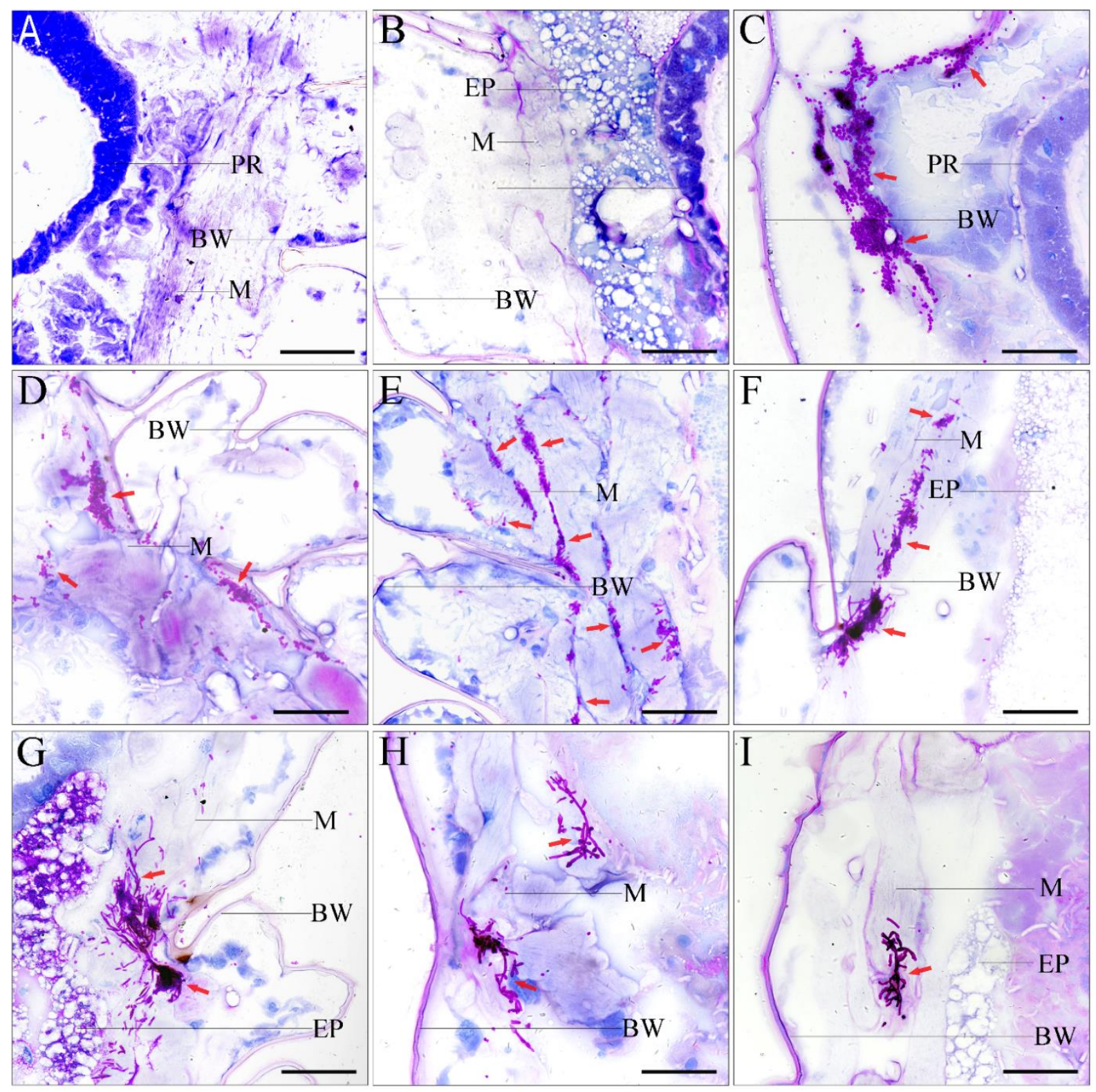

Figure 3 Histopathological identification of $C$. albicans in housefly larvae (PAS stain, $\times 200$ ). A Blank control group: untreated larvae. B PBS negative control: larvae were injected with equal volume PBS buffer. C-I Infection group: larvae were injected with C. albicans larvae. C 3 h post-infection (PI), D 6 h PI, E 12 h PI, F 18 h PI, G 24 h PI, H 36 h PI and I 48 h PI. Proventriculus (PR), body wall (BW), epiploon (EP), muscle (M), arrows indicate C. albicans. Bar $=100 \mu \mathrm{m}$

In all infected larvae, many scattered brown spots were observed. These spots were dark in colour because of the accumulation of melanin. Another plane of histological sections revealed that these spots were comprising melanized encapsulation and encapsulated agglutination of fungal cells, spread among the coelom, muscle tissue and the inner side of the body wall (Fig. 4). Between 3 and $12 \mathrm{~h} \mathrm{PI}$, we observed a considerable number of $C$. albicans with yeast forms aggregated around the melanized encapsulation (Figs. 4A, 4B and 4C), the yeast and hyphal forms were both present between 18 and $24 \mathrm{~h}$ (Figs. 4D and 4E). C. albicans majority existed as hyphal forms at $36 \mathrm{~h}$ and $48 \mathrm{~h}$ (Figs. 4F and 4G), and some empty capsules without fungal cellss were found at $48 \mathrm{~h} \mathrm{PI}$ (Figs. $4 \mathrm{H}$ and $4 \mathrm{I}$ ). 

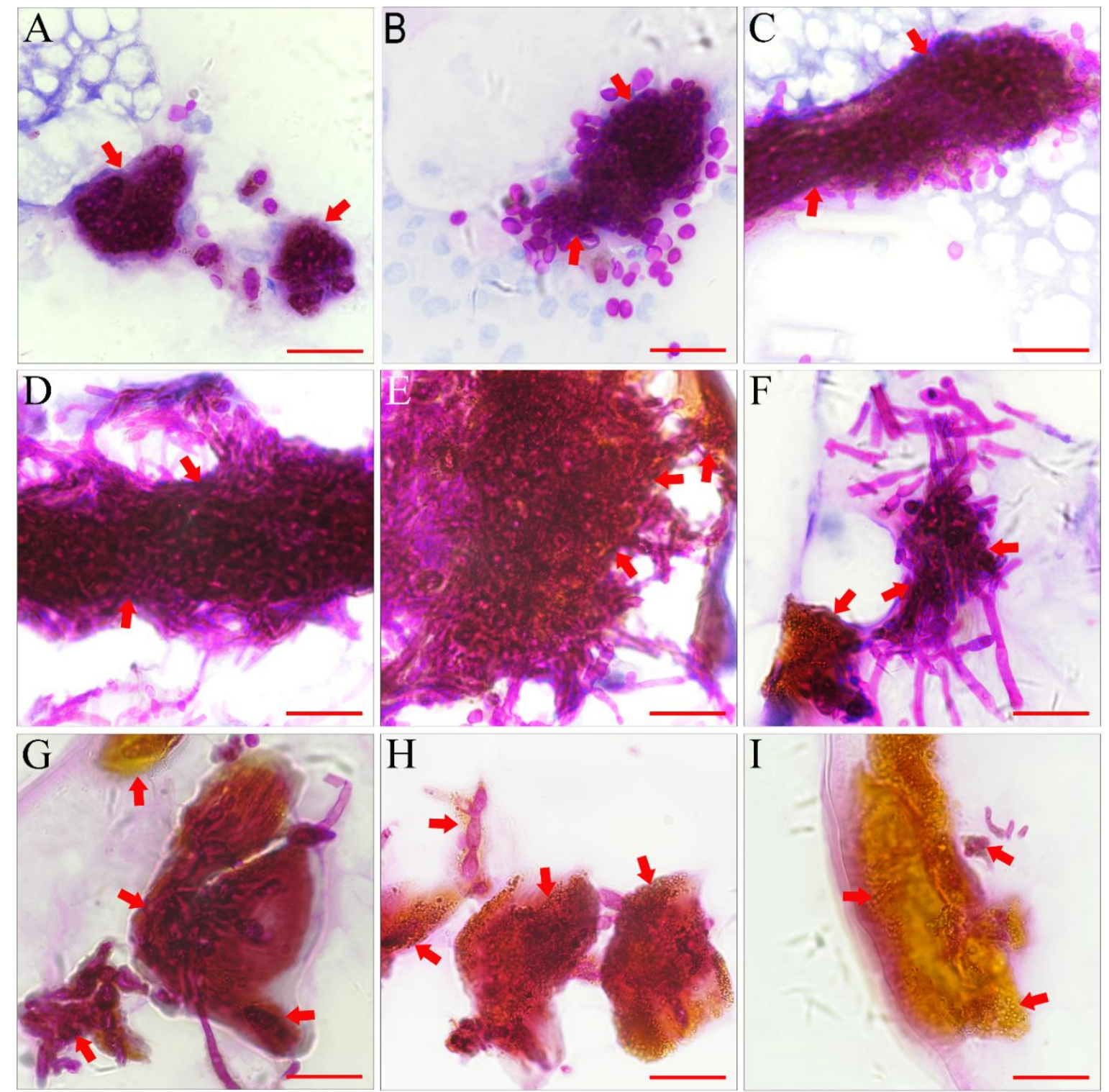

Figure 4 Histopathological identification of melanized encapsulations. A $3 \mathrm{~h}$ post-infection (PI), melanized encapsulations encysted yeast from fungal cells. B 6 h PI similar to 3 h PI. C At 12 h PI, melanized encapsulations encysted yeast from fungal cells. D At $18 \mathrm{~h} \mathrm{PI}$, melanized encapsulations encysted yeast and hyphae from fungal cells. E 24 h PI similar to 18 h PI. F At 36 h PI, melanized encapsulations encysted hyphae from fungal cells. G At 48 h PI, melanized encapsulations encysted hyphae from fungal cells. H At 48 h PI, melanized encapsulations encysted limited amount of hyphae from fungal cells. I At 48 h PI, empty encapsulations. Arrows indicate melanized encapsulations. Bar $=50 \mu \mathrm{m}$

\section{qPCR analyses of AMP genes expression.}

qPCR was used to assay the temporal patterns of mRNA from attacin, cecropin, defensin and diptericin at respectively challenged 3rd-instar larva at $3,6,12,18,24,36$ and $48 \mathrm{~h}$ after $C$. albicans infection. Comparing with the PBS group, the results revealed that in coordination with the variation during the infection process, the expression levels of four antimicrobial peptides were up-regulated, but their expression patterns were different from each other (Fig. 5). Attacin, cecropin and defensin were gradually increased at 3 h PI, but diptericin was not clearly up-regulated $(P$ $>0.05$ ). Between 6 and $24 \mathrm{~h}$ PI, all four AMPs were significantly up-regulated $(P<0.05$, in all cases). Expression level of attacin, cecropin peaked at $12 \mathrm{~h}$ PI, denfensin peaked at $3 \mathrm{~h}$ PI and diptericin peaked at $24 \mathrm{~h} \mathrm{PI}$. And then from 36 to $48 \mathrm{~h} \mathrm{PI}$, expression level of attacin, defensin and diptericin sharply decreased $(P>0.05$, in all cases $)$, and cecropin 
expression level slowly decreased $(P<0.05$, in all cases).

A

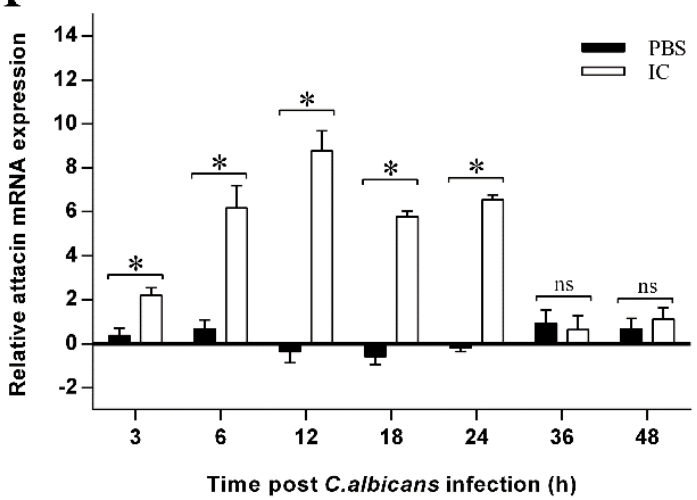

$\mathrm{C}$

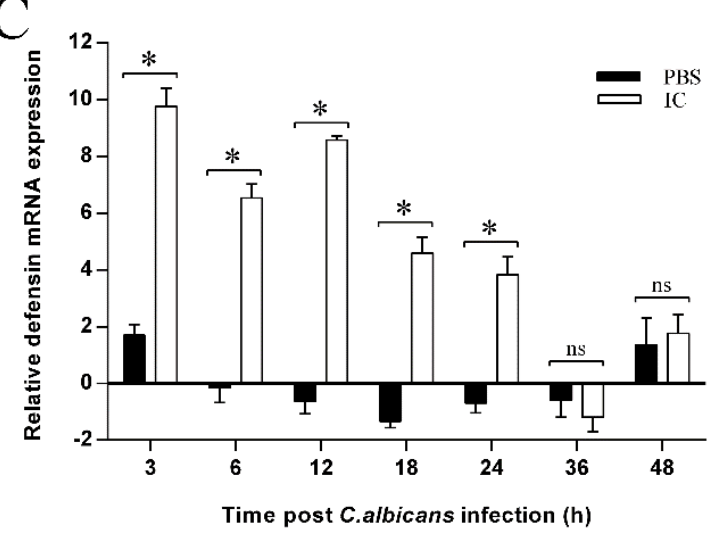

B

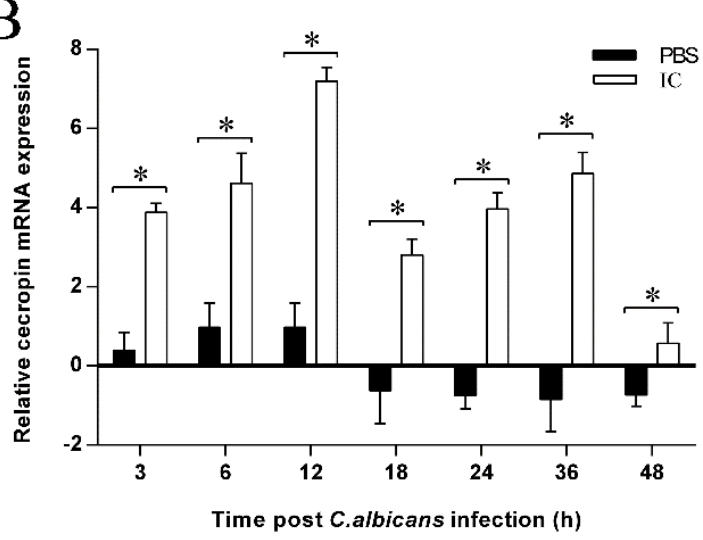

$\mathrm{D}$

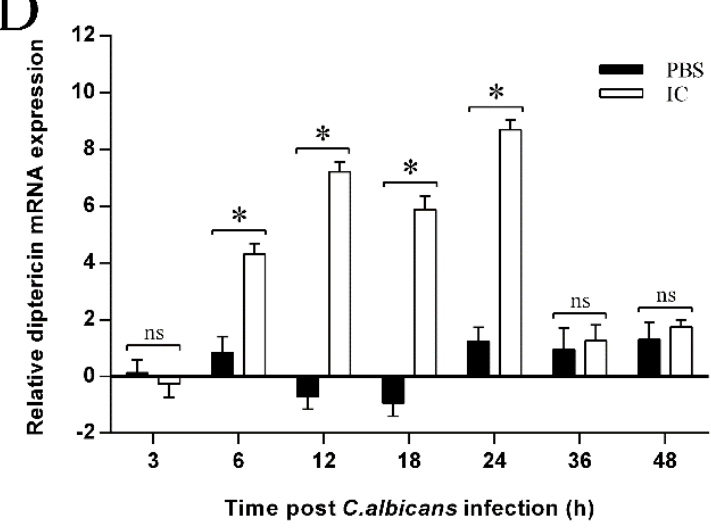

Figure 5 AMP genes expression pattern in housefly larvae with $C$. albicans infection. In the IC group, the larvae were injected with $210 \mathrm{nl}$ suspension of $C$. albicans $\left(2 \times 10^{4} \mathrm{CFU}\right)$. In the PBS group, the larvae were injected with equal volume PBS buffer. The $\mathrm{C}$ group was untreated. Collecting samples ( $n=4$ larvae per time point) at $3,6,12,18,24$, 36 and $48 \mathrm{~h}$ post-infection. The $\mathrm{C}$ group was set as calibrator conditions, compared with the PBS and IC groups. For relative expression analysis, CT values for calibrator conditions (i.e. larvae untreated at each indicated time points, $\mathrm{C}$ group) and treatment groups (i.e. PBS and IC groups) were calculated using the $2^{-\Delta \Delta \mathrm{CT}}$ method. $\log _{2}$-fold change in expression ratios of the target genes attacin, cecropin, defensin and diptericin was compared with the calibrator conditions using the reference gene rps18. A attacin, $\mathbf{B}$ cecropin, $\mathbf{C}$ defensin, $\mathbf{D}$ diptericin. Data was presented as mean \pm SE. Significance levels: $*$ P $<0.05 ;$ ns, not significant

\section{DISCUSSION}

Insects lack the adaptive immune responses typical of vertebrates; thus, they are heavily dependent on their innate immune system to defend against pathogenic invasion. Knowledge on how the insect innate immune response fights infection may aid the understanding of the first step in the human host-pathogen interaction (Wang et al. 2013; Singh et al. 2014; Wu et al. 2014; Khalil et al. 2015). Many models and techniques of insect infection have been successfully established, including injury, rolling and ingestion methods. The rolling method involves the infection of the epithelial cell surface and the ingestion method involves the infection of the digestive tract mucous membrane. These methods model the natural infectious process of pathogenic invasion into the epidermis and intestinal tract, which can then cause local infection that may gradually develop into systemic infection (Onfelt et al. 2001; Nayduch et al. 2013; Wu et al. 2014). The injury method mainly used needles to deliver pathogens by piercing the somatic layer or directly injects pathogens into the insect coelom to cause acute infection and rapidly activate the immune response (Matsumoto et al. 2012; Singh et al. 2014). However, the inevitable mechanical injury caused by this method may affect the experimental results. Micro-injection technology has recently been applied in $D$. melanogaster 
infection to achieve controllable infection and minimize mechanical injury by a precise and quantitative inoculation (Khalil et al. 2015). In our preliminary study, the third-instar housefly larvae infection model was built using minimally invasive injection methods. The previous results have indicated that all the larvae of the PBS group were able to complete their life cycle, and in the meanwhile, the larvae of the IC group were controlled with the ratio of approximately $50 \%$. In this study, morphological and histological investigation revealed no significant difference between larvae that were not inoculated and those that were inoculated with PBS (Figs.1 and 2). Although the qPCR results revealed a slight difference in AMP expression between the PBS and $\mathrm{C}$ groups, no significant differences were observed. This fluctuation may have been caused by the activation of a weak immune response by the micro wound on the larvae somatic layer caused by injection.

We observed that antibacterial peptides were synthesized in response to microorganism infestation. The housefly genome contains more antibacterial peptide genes than other insect genomes, and such genes are present in the form of a gene family in the genome (Scott et al. 2014; Tang et al. 2014). It was previously reported that in houseflies challenged by microorganisms, antibacterial peptides such as attacins, cecropins, defensins and diptericins were significantly upregulated. These rapid changes in expression were coordinated in several genes (Wang et al. 2009; Liu et al. 2011; Fleming et al. 2014). In this study, all four antibacterial peptides (attacins, cecropins, defensins and diptericins) were simultaneously upregulated 6-24 h PI, and their expression parrterns were consistent with those of our previous experiments and some other previouslty published manuscripts (Wang et al. 2009; Fleming et al. 2014). In D. melanogaster AMP expression is regulated by two signal transduction pathways: the Toll and IMD signal pathways. The Toll pathway regulates the response to gram-positive bacterial and fungal infections. The IMD pathway regulates the response to gram-negative bacterial infection (Cho et al. 2012; Kleino and Silverman 2014). Even though the Toll and IMD pathways have separate components and preferential target genes, but some antimicrobial peptide are expressed following the stimulation of one of the two aforementioned pathways (Hultmark 2003; Brennan et al. 2004, Royet et al. 2005, Tanji et al. 2005). We found that the expression of attacin, cecropin, defensin and diptericin varied 3 to $48 \mathrm{~h}$ PI. For instance, cecropin and defensin were up-regulated at $3 \mathrm{~h} \mathrm{PI}$, whereas attacin and diptercin were up-regulated later, and in particular, diptercin was not up-regulated until $6 \mathrm{~h}$ PI. The rapid response of cecropin and defensin suggested that because houseflies were infected with $C$. albicans, they may preferentially respond to the Toll pathway. Other studies have shown that the Toll and IMD pathways can be cooperative and may contribute to the activation of each other (Lemaitre and Hoffmann 2007; Tanji et al. 2007, Xiong et al. 2015). Defensin and cecropin are predominantly regulated by the Toll pathway, and attacin and diptericin are predominantly regulated by the IMD pathway during the $C$. albicans infection. Cecropin is reported to possess fungistasis activity in vitro (Hultmark 2003); therefore, the maintenance of high cecropin expression throughout infection process may have contributed to the control of infection.

Melanization is another major component of arthropod immunity that is not observed in vertebrates. Melanization involves the rapid synthesis of a black pigment, melanin, at the site of infection or injury (Cerenius et al. 2008; Tang 2009; Dudzic et al. 2015). Melanized encapsulation was reported to form at the site at which pathogens infected insects and are activated by contact between the pathogen and hemolymph cells (e.g. granular cells). Hemolymph cells then release chemotactic components to absorb plasma cells and form a multicellular sheet. The encapsulation internal layer undergoes melanization to form a capsule around the pathogen through the action of phenoloxidase (Lavine and Strand 2002; Lu 2008), and the melanization cascade activates the host humoral immune defence system, as observed in Legionella pneumophila infection of G. mellonella (Harding et al. 2012). Other research showed that Silkworm serine protease homologs (e.g. SPH1 and SPH2) and prophenoloxidase (e.g. BmPO1) were involved in melanization and encapsulation (Tokura et al. 2014). Moreover, Wang reported that lectins played a role in the coordination of hemagglutination, encapsulation and melanization in Helicoverpa armigera pathogen elimination (Wang et al. 2014). Housefly larvae infected with $E$. coli are reported to eliminate pathogens by activating the phenoloxidase cascade ( $\mathrm{Li}$ et al. 2015), which enhances hemagglutination and promotes cytophagocytosis through lectin (Cao et al. 2012). In this study, we observed brown spots 
under the epidermis of larvae within $3 \mathrm{~h}$ of infection with $C$. albicans. The brown spots were Melanized encapsulations surrounding aggregated $C$. albicans mycothallus. Throughout infection, similar structures were found, and some empty encapsulations without mycothallus were found 48 h PI. Our results suggested that encapsulation and agglutination may play an important role in the housefly innate immune response against $C$. albicans.

Housefly larvae, like some other insects, have a mixocoel, and humoral hemocyte concentrated in the last segments and scattered around tissues and organs. In this study, C. albicans mycothallus colonized in the gaps between the body wall and the muscle layer, as well as among muscle fibers of the muscle layer of larvae. We speculated that $C$. albicans was injected into the hemocoel at the 10th segment of the segmental venter and was quickly disseminated through the blood stream during muscle contractions to the entire body. C. albicans was able to persist between the inner side of the body wall and muscle tissue layers because of the low concentration of hemocytes in these spaces. In these environments, $C$. albicans was able to develop from the yeast to hyphal form (Huang 2012; Mayer et al. 2013). Our data are consistent with previous research reporting the formation of pseudomycelium by Candida tropicalis mycothallus when hemocyte numbers were reduced in G. mellonella (Mesa-Arango et al. 2013). Some reports suggested that insect blood cells were constantly regenerated and kept dynamically changing. In Locusta migratoria, the hemocytes and hematopoietic tissue mutually assist each other to clear invading pathogens from the circulation (Duressa et al. 2015). In housefly, hemocyte number varied during fungal infection (Mishra et al. 2015).

According to these results, we infer that because a great deal of fungal spores was injected into the coelom of housefly larvae, a fraction was transported to the gaps of tissues. Because there are fewer hemocytes in these locations than in the haemocoel, fungus which stayed in these locations was not eliminated immediately by the immune system. And then began to colonize at these locations. With the regeneration hemocytes and other immune molecule such as AMPs were transported to these gaps during blood circulation, these rest fungus were gradually cleared. This may explain why the mycothallus distribution changed from a continuous line to scattered spots and then disappeared.

The immune response should be carefully viewed as a double edged sword. Although it protected the larvae from pathogen invasion, excessive immune response could also cause self injury. Similar to the vertebrate immune system, the insect immune system is also regulated by complicated control mechanisms (Cerenius et al. 2008). A great number of immune regulators are involved in these processes, including serine proteinase inhibitors (Serpin). In Tenebrio molitor (Jiang et al. 2009) and D. melanogaster (Ligoxygakis et al. 2002; Fullaondo et al. 2011), serpins can regulate the immune response through the Toll signal pathway and prophenoloxidase-activating system. $\mathrm{Li}$ discovered at least 11 serpins in housefly, including a gene homologous to serpin27A of $D$. melanogaster ( $\mathrm{Li}$ et al. 2015). In this study, at $48 \mathrm{~h}$ PI, the scattered brown spots gradually disappeared and empty Melanized encapsulations were observed and mycothallus colonization and AMP expression were reduced, indicating that mechanisms negatively regulating the immune response had begun to play their roles following the elimination of C. albicans. At 3 and 48 h PI, the expression level of 4 AMPs experienced upregulated and then gradual recovery suggested that the balance, which provides protection for developing insects, permitting qualitatively normal inflammatory responses and protection against infection, occurred in Musca domestica as it did in vertebrates. However, the mechanisms underlying melanized encapsulation, coagulation and AMP expression during $C$. albicans infection remain to be determined. Thus, further study into fungal infection of the housefly is warranted.

\section{CONCLUSIONS}

In conclusion, we studied the morphology and histology of $C$. albicans infected M. Domestica larvae between 3 and $48 \mathrm{~h}$ PI. The infection involved a series of stages, including injection, infection, immune response and elimination of the fungal pathogen, and the housefly immune response was observed to involve the up-regulation of AMP, melanized encapsulation and agglutination.

\section{ACKNOWLEDGMENTS}

We thank Hui Xun, Rong Mou, Jia-Hong Wu and Bo Zhou for their technical expertise, Zhu Zeng for his 
critical review of this manuscript. All experiments in this study were performed in the Modern Pathogenic Biology Laboratory and the Center for basic medical science of Guizhou Medical University. This study was funded by National Natural Science Foundation of China (81360254, 81560337), National Science and technology support program Foundation of China (2011BAC06B12) and Science and Technology Foundation of Guizhou Province (LH [2014]7076, gzwjkj2014-2-100).

\section{REFERENCES}

Altincicek B, Knorr E, Vilcinskas A. Beetle immunity: identification of immune-inducible genes from the model insect Tribolium castaneum. Dev Comp Immunol. 2008; 32:585-595.

Brennan CA, Anderson KV. Drosophila: the genetics of innate immune recognition and response. Annu Rev Immunol. 2004; 22:457-483.

Cao XH, Zhou MH, Wang CL, Hou LH, Li YY, Chen LY. Musca domestica pupae lectin improves the immunomodulatory activity of macrophages by activating nuclear factor-kappa B. J Med Food. 2012; 15:145-151.

Cerenius L, Lee BL, Soderhall K. The proPO-system: pros and cons for its role in invertebrate immunity. Trends Immunol. 2008; 29:263-271.

Chamilos G, Samonis G, Kontoyiannis, DP. Drosophila melanogaster as a model host for the study of microbial pathogenicity and the discovery of novel antimicrobial compounds. Curr Pharm Design. 2011; 17:1246-1253

Cho IH, Jeon JW, Paek SH, Kim DH, Shin HS, Ha UH, et al. Toll-like receptor-based immuno-analysis of pathogenic microorganisms. Anal Chem. 2012; 84:9713-9720.

Cirimotich CM, Ramirez JL, Dimopoulos G. Native microbiota shape insect vector competence for human pathogens. Cell Host Microbe. 2011; 10:307310.

Dang XL, Wang YS, Huang YD, Yu XQ, Zhang WQ. Purification and characterization of an antimicrobial peptide, insect defensin, from immunized house fly (Diptera: Muscidae). J Med Entomol. 2010; 47:11411145.

Duressa TF, Vanlaer R, Huybrechts R. Locust cellular defense against infections: sites of pathogen clearance and hemocyte proliferation. Dev Comp Immunol. 2015; 48:244-253.

Dudzic JP, Kondo S, Ueda R, Bergman CM, Lemaitre B. Drosophila innate immunity: regional and functional specialization of prophenoloxidases. BMC Biol. 2015; $13: 81$.

$\mathrm{Fu} \mathrm{P}, \mathrm{Wu}$ JW, Guo G. Purification and molecular identification of an antifungal peptide from the hemolymph of Musca domestica (housefly). Cell Mol Immunol. 2009; 6:245-251. doi: 10.1038/cmi.2009.33

Fu P, Wu JW, Gao S, Guo G, Zhang Y, Liu JThe recombinant expression and activity detection of MAF-1 fusion protein. Sci Rep-Uk. 2015; 5:14716.

Fleming A, Kumar HV, Joyner C, Reynolds A, Nayduch D. Temporospatial fate of bacteria and immune effector expression in house flies fed GFPEscherichia coli O157: H7. Med Vet Entomol. 2014; 28:364-371.

Fuchs BB, Eby J, Nobile CJ, El Khoury JB, Mitchell AP, Mylonakis E. Role of filamentation in Galleria mellonella killing by Candida albicans. Microbes Infect. 2010; 12:488-496.

Fullaondo A, Garcia-Sanchez S, Sanz-Parra A, Recio E, Lee SY, Gubb D. Spn1 regulates the GNBP3dependent Toll signaling pathway in Drosophila melanogaster. Mol Cell Biol. 2011; 31:2960-2972.

Harding CR, Schroeder GN, Reynolds S, Kosta A, Collins JW, Mousnier A, Frankel G. Legionella pneumophila pathogenesis in the Galleria mellonella infection model. Infect Immun. 2012; 80:2780-2790.

Hoffmann JA. The immune response of Drosophila. Nature. 2003; 426:33-38.

Huang GH. Regulation of phenotypic transitions in the fungal pathogen Candida albicans. Virulence. 2012; 3:251-261.

Hultmark D. Drosophila immunity: paths and patterns. Curr Opin Immunol.2003; 15:12-19.

Jiang R, Kim EH, Gong JH, Kwon HM, Kim CH, Ryu $\mathrm{KH}$, et al. Three pairs of protease-serpin complexes cooperatively regulate the insect innate immune responses. J Biol Chem. 2009; 284:35652-35658.

Kelly J, Kavanagh K. Caspofungin primes the immune response of the larvae of Galleria mellonella and induces a non-specific antimicrobial response. $\mathrm{J} \mathrm{Med}$ Microbiol. 2011; 60: 189-196.

Khalil S, Jacobson E, Chambers MC, Lazzaro BP. Systemic bacterial infection and immune defense phenotypes in Drosophila melanogaster. Jove-J Vis Exp. 2015; 99:e52613.

Kleino A, Silverman N. The Drosophila IMD pathway in the kactivation of the humoral immune response. Dev Comp Immunol. 2014; 42:25-35.

Lavine MD, Strand MR. Insect hemocytes and their role in immunity. Insect Biochem Molec. 2002; 32:12951309.

Lemaitre B, Hoffmann J. The host defense of Drosophila melanogaster. Апnu Rev Immunol. 2007; 25:697743.

Li DX, Liang YL, Wang XW, Wang L, Qi M, Yu Y, Luan YY. Transcriptomic analysis of Musca domestica to reveal key genes of the Prophenoloxidase-activating system. G3-Genes Genom Genet. 2015; 5:1827-1841.

Liang YL, Wang JX, Zhao XF, Du XJ, Xue JF. Molecular cloning and characterization of cecropin 
from the housefly (Musca domestica), and its expression in Escherichia coli. Dev Comp Immunol. 2006; 30:249-257.

Ligoxygakis P, Pelte N, Ji CY, Leclerc V, Duvic B, Belvin $\mathrm{M}$, et al. A serpin mutant links Toll activation to melanization in the host defence of Drosophila. Embo J. 2002; 21:6330-6337.

Liu FS, Sun LL, Tang T, Wang LN. Cloning, sequence analysis and induced expression of attacin-2 gene in housefly (Musca domestica). Acta Entomologica Sinica. 2011; 54:27-33. Chinses.

Lu HS. Principles of insect immunology. Shanghai Scientific and Technical Publishers, Shanghai, China; 2008. Chinses.

Matsumoto Y, Miyazaki S, Fukunaga DH, Shimizu K, Kawamoto S, Sekimizu K. Quantitative evaluation of cryptococcal pathogenesis and antifungal drugs using a silkworm infection model with Cryptococcus neoformans. J Appl Microbiol. 2012; 112:138-146.

Mayer FL, Wilson D, Hube B. Candida albicans pathogenicity mechanisms. Virulence. 2013; 4:119128.

Mesa-Arango AC, Forastiero A, Bernal-Martinez L, Cuenca-Estrella M, Mellado E, Zaragoza O. The nonmammalian host Galleria mellonella can be used to study the virulence of the fungal pathogen Candida tropicalis and the efficacy of antifungal drugs during infection by this pathogenic yeast. Med Mycol. 2013; 51:461-472.

Miceli MH, Diaz JA, Lee SA. Emerging opportunistic yeast infections. Lancet Infect Dis. 2011; 11:142151.

Mishra S, Kumar P, Malik A. The effect of Beauveria bassiana infection on cell mediated and humoral immune response in house fly, Musca domestica L. Environ Sci Pollut R. 2015; 22:15171-15178.

Nayduch D, Cho H, Joyner C. Staphylococcus aureus in the house fly: temporospatial fate of bacteria and expression of the antimicrobial peptide defensin. $J$ Med Entomol. 2013; 50:171-178.

Okada M, Hisajima T, Ishibashi H, Miyasaka T, Abe S, Satoh T. Pathological analysis of the Candida albicans-infected tongue tissues of a murine oral candidiasis model in the early infection stage.[J]. Arch Oral Biol. 2013; 58:444-450.

Onfelt Tingvall T, Roos E, Engstrom Y. The IMD gene is required for local cecropin expression in Drosophila barrier epithelia. Embo Rep. 2001; 2:239243.

Pfaffl MW, Horgan GW, Dempfle L. Relative expression software tool (REST) for group-wise comparison and statistical analysis of relative expression results in real-time PCR. Nucleic Acids Res. 2002; 30:e36.

Pei ZH, Sun XN, Tang Y, Wang K, Gao YH, Ma HX. Cloning, expression, and purification of a new antimicrobial peptide gene from Musca domestica larva. Gene. 2014; 549:41-45.
Phoku JZ, Barnard TG, Potgieter N, Dutton MF. Fungi in housefly (Musca domestica L.) as a disease risk indicator-A case study in South Africa. Acta Trop. 2014; 140:158-165.

Royet J, Reichhart JM, Hoffmann. Sensing and signaling during infection in Drosophila. Curr Opin Immunol. 2005; 17:11-17.

Scott JG, Warren WC, Beukeboom LW, Bopp D, Clark AG, Giers SD, et al. Genome of the house fly, Musca domestica L., a global vector of diseases with adaptations to a septic environment. Genome Biol. 2014; $15: 466$.

Singh S, Reese JM, Casanova-Torres AM, GoodrichBlair H, Forst S. Microbial population dynamics in the hemolymph of Manduca sexta infected with Xenorhabdus nematophila and the entomopathogenic nematode Steinernema carpocapsae. Appl Environ Microb. 2014; 80:42774285 .

Stokes BA, Yadav S, Shokal U, Smith LC, Eleftherianos I. Bacterial and fungal pattern recognition receptors in homologous innate signaling pathways of insects and mammals. Frint Microbiol. 2015; 6:19.

Tang T, Li X, Yang X, Yu X, Wang JH, Liu F, Huang DW. Transcriptional response of Musca domestica larvae to bacterial infection. Plos One. 2014; 9:e104867.

Tang HP. Regulation and function of the melanization reaction in Drosophila. Fly. 2009; 80:105-111.

Tanji T, Hu XD, Weber ANR, Ip YT. Toll and IMD pathways synergistically activate an innate immune response in Drosophila melanogaster. Mol Cell Biol. 2007; 27:4578-4588.

Tanji T, Ip YT. Regulators of the Toll and Imd pathways in the Drosophila innate immune response. Trends Immunol. 2005; 26:193-198.

Tokura A, Fu GS, Sakamoto M, Endo H, Tanaka S, Kikuta S, et al. Factors functioning in nodule melanization of insects and their mechanisms of accumulation in nodules. J Insect physiol. 2014; 60:40-49.

Wang JL, Zhang Q, Tang L, Chen L, Liu XS, Wang YF. Involvement of a pattern recognition receptor C-type lectin 7 in enhancing cellular encapsulation and melanization and melanization due to its carboxylterminal CRD domain in the cotton bollwork, Helicoverpa armigera. Dev Comp Immunol. 2014; 44:21-29.

Wang JX, Zhao XF, Liang YL, Li L, Zhang W, Ren Q, et al. Molecular characterization and expression of the antimicrobial peptide defensin from the housefly (Musca domestica). Cell Mol Life Sci. 2006; 63:3072-3082.

Wang Y, Jin XB, Zhu JY, Zeng AH, Chu FJ, Yang XR, et al. Expression pattern of antibacterial genes in the Musca domestica. Sci China Ser C. 2009; 52:823830. 
Wang Y, Li DD, Jiang YY, Mylonakis E. Utility of Insects for Studying Human Pathogens and Evaluating New Antimicrobial Agents. In: Vilcinskas A, editor. Yellow biotechnology I: insect biotechnologie in drug discovery and preclinical research. Springer-Verlag Berlin; 2013. pp 1-25.

Wu GQ, Li M, Liu Y, Ding Y, Yi YH. The specificity of immune priming in silkworm, Bombyx mori, is mediated by the phagocytic ability of granular cells. J Insect Physiol. 2015; 81:60-68.

Wu MH, Sugimura Y, Iwata KV, Takaya N, Takamatsu $\mathrm{D}$, Kobayashi $\mathrm{M}$, et al. Inhibitory effect of gut bacteria from the Japanese honey bee, Apis cerana japonica, against Melissococcus plutonius, the causal agent of European foulbrood disease. J Insect Sci. 2014; 14:129.
Xiong GH, Xing LS, Lin Z, Saha TT, W CS, Jiang HB et al. High throughput profiling of the cotton bollworm Helicoverpa armigera immunotranscriptome during the fungal and bacterial infections. BMC Genomics. 2015; 16:321.

Yang X, Tang T, W YL, Liu X, Cao XR, N ZH, et al. Gene cloning, expression profiling and antimicrobial assay of Muscin, a new antimicrobial peptide in the housefly (Musca domestica). Acta Entomologica Sinica. 2015; 58:617-624. Chinses.

Ohta M, Watanabe A, Mikami T, Nakajima Y, Kitaini M, Tabunoki H, et al. Mechanism by which Bombyx mori hemocytes recognize microorganisms: direct and indirect recognition systems for PAMPs. Dev Comp Imm 